
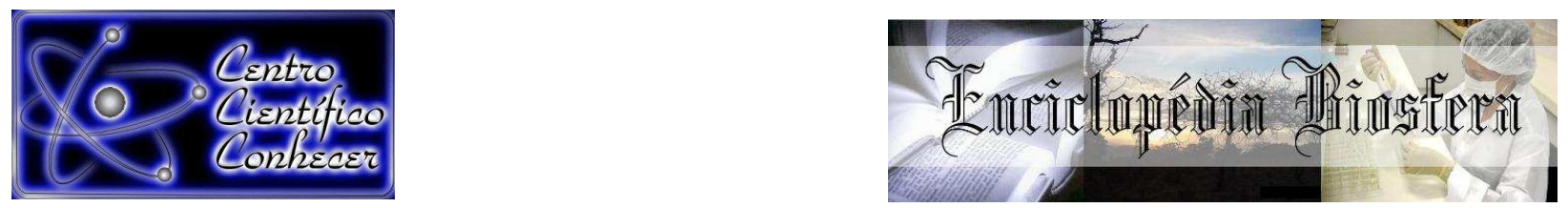

\title{
ANÁLISE DA PROBABILIDADE PARA TRANSTORNO MENTAL COMUM ENTRE CUIDADORES DE IDOSOS DO SUDESTE GOIANO
}

Kamylla Guedes de Sena ${ }^{1^{*}}$, Luiz Henrique Batista Monteiro², Ivânia Vera ${ }^{3}$, Roselma Lucchese $^{4}$, Moisés Fernandes Lemos ${ }^{5}$

1-Graduanda em Enfermagem, Universidade Federal de Goiás (UFG), Regional Catalão. Catalão-GO, Brasil. (kamylla_g.s@hotmail.com)

2-Graduando em Enfermagem, Universidade Federal de Goiás (UFG), Regional Catalão. Catalão-GO, Brasil.

3-Docente do Curso de Enfermagem, Universidade Federal de Goiás (UFG), Regional Catalão. Catalão-GO, Brasil.

4-Docente do Curso de Enfermagem, Universidade Federal de Goiás (UFG), Regional Catalão. Catalão-GO, Brasil.

5-Docente do Curso de Psicologia, Universidade Federal de Goiás (UFG), Regional Catalão. Catalão-GO, Brasil.

Recebido em: 08/04/2017 - Aprovado em: 10/06/2017 - Publicado em: 20/06/2017 DOI: 10.18677/EnciBio_2017A120

\section{RESUMO}

As doenças psíquicas representam de um terço a $14 \%$ de morbidades em escala mundial, na qual o sexo feminino apresenta maior taxa para Transtorno Mental Comum (TMC) ao se equiparar ao sexo masculino. Um instrumento que tem se mostrado eficaz no rastreamento do TMC é o Self Report Questionnaire (SRQ-20) aplicado em diversos grupos populacionais, sendo amplamente utilizados em pesquisas da área da saúde. Assim este estudo objetivou descrever a prevalência de TMC entre cuidadores de idosos. Tratou-se de uma pesquisa de corte transversal, a coleta de dados foi desenvolvida em uma ação de extensão, no mês de dezembro de 2015. Dentre os 14 cuidadores de idosos que compõe a amostra do presente estudo, constatou-se a predominância de idade entre 41-50 anos (57,1\%); sexo feminino (92,9\%); vivendo sem companheiro $(57,1 \%)$; nível de escolaridade até 8 anos $(57,1 \%)$ e média de TMC encontrada nesta pesquisa foi de 3.78 , representando $28.6 \%$ da amostra de acordo com o SRQ 20. Evidencia-se ainda uma elevada probabilidade de desenvolvimento de TMC entre o sexo feminino em comparação ao masculino, fazendo necessário a criação e implementação de políticas públicas que garantam a qualidade na atenção a saúde e qualidade de vida da pessoa idosa, bem como de seu cuidador.

PALAVRAS-CHAVE: Cuidadores, Idoso, Saúde Mental.

\section{ANALYSIS OF PROBABILITY FOR COMMON MENTAL DISORDER AMONG ELDERLY CAREGIVERS OF SOUTHEAST GOIANO}

\section{ABSTRACT}

The psychic diseases represent one-third to $14 \%$ of morbidities in the global scala, in which the female sex has a higher rate for Common Mental Disorder (CMD) to equal the male. An instrument that has proven effective in tracking the TMC is the Self Report Questionnaire (SRQ-20) applied in the various population ENCICLOPÉDIA BIOSFERA, Centro Científico Conhecer - Goiânia, v.14 n.25; p.1444 2017 
groups, being widely used in research in the area of health. Thus this study aimed to describe the prevalence of CMD among elderly caregivers. It was a cross-sectional survey, the data collection was developed in an action of extension, in the month of December 2015. Among the 14 caregivers of elderly that makes up the sample of this study, it was verified the prevalence of aged 41-50 years $(57.1 \%)$; female (92.9\%); living without a partner $(57.1 \%)$; the level of schooling up to 8 years $(57.1 \%)$ and the average CMD found in this research was 3.78 , representing $28.6 \%$ of the sample according to the SRQ 20 . This evidence a high probability of the development of CMD among the female compared to male, making necessary the creation and implementation of public policies that ensure the quality in the attention to health and quality of life of the old person, as well as the caregiver.

KEYWORDS: Caregivers, Aged, Mental Health.

\section{INTRODUÇÃO}

As doenças psíquicas representam de um terço a $14 \%$ de morbidades em escala mundial (PRINCE et al., 2007; SKAPINAKIS et al., 2013). Em uma metanálise, observou-se que a prevalência mundial para Transtorno Mental Comum (TMC) é de $29,2 \%$, sendo que as mulheres são mais susceptíveis para o desenvolvimento dessa patologia (STEEL et al., 2014). O sexo feminino apresenta maior taxa para TMC (19.8\%) ao se equiparar ao sexo masculino $(15,2 \%)$ (ANGST et al., 2015).

O TMC corresponde aos transtornos a seguir, com as classificações baseadas na Classificação Internacional de Doenças (CID-10): transtorno de ansiedade generalizada (CID-F41.1), do pânico (CID-F41.0), de ansiedade social (CID-40.1), obsessivo-compulsivo (CID-F42.2), de estresse pós-traumático (CIDF43.1), depressão (CID- F32.0) e fobias (CID-40.2) (BESSANT \& EKERS, 2011; OMS, 1997).

No contexto do TMC, nota-se maior prevalência para depressão e ansiedade, sendo que estas variam de acordo o espaço geográfico e condições de vida e saúde dos indivíduos. Nesse ínterim, tem-se respectivamente as seguintes prevalências para depressão e ansiedade $73.6 \%$ e $81.8 \%$ no continente europeu, 49.8\% e 69.7\% na América Latina, $47.0 \%$ e $43.7 \%$ ao norte do continente africano e 14.5 e 15.5\% no continente asiático (BAXTER et al., 2013).

Em relação aos cuidadores de idosos, a prevalência de TMC é de 26,7\% (GRATÃO et al., 2015). Vale salientar que tal prevalência dar-se-á em relação aos sentimentos e preocupações que os cuidadores dos idosos expressam, particularmente a tangencialidade e a especificidade do cuidado contínuo, ao considerar a condição física e incapacidade do idoso, o convívio diário, e a dependência do idoso (BATISTA \& ARAÚJO, 2011; ROSA et al., 2011; GRATÃO et al., 2013).

Um instrumento eficaz no rastreamento do TMC é o Self Report Questionnaire (SRQ-20) aplicado em diversos grupos populacionais, sendo amplamente utilizado em pesquisas da área da saúde. O SRQ-20 investiga a existência de sintomas de suspeita de um transtorno mental, este oportuniza a identificação precoce de uma desordem mental, porém não elimina a necessidade de um diagnóstico específico (MOREIRA et al., 2011).

$O$ instrumento foi criado (HARDING et al., 1980) e validado no Brasil em 1986 (MARI \& WILLIANS, 1986) sendo reorganizado em 2008 considerando as características psicométicas, emocionais e de saúde mental da comunidade, para 
o monitoramento de TMC (MARI \& WILLIANS, 1986; GONÇALVES et al., 2008). Com vistas à atenção integral e a melhoria da qualidade da assistência ofertada aos idosos, por parte dos cuidadores e, por considerar todos os aspectos citados, este estudo objetivou descrever a prevalência de TMC entre cuidadores de idosos.

Tipo e local do estudo

\section{MATERIAL E MÉTODOS}

Tratou-se de uma pesquisa de corte transversal. Caracterizada por um estudo planejado de uma determinada população em um momento pontual, este tipo de estudo é amplamente utilizado por pesquisadores na área da saúde (MEDRONHO et al., 2009). A população pesquisada consistiu em cuidadores de idosos, que atuam em um município do sudeste goiano, na região Brasil Central. A pesquisa em questão pertence a um projeto de pesquisa matriz, intitulado Análise em Saúde Mental e constituição da rede no Sistema Único de Saúde no Sudeste Goiano, CAAE nำ25586013.2.0000.5083.

\section{Coleta de dados}

A coleta de dados foi desenvolvida em uma ação de extensão, no mês de dezembro de 2015, com uma amostra de 14 cuidadores. Os cuidadores foram convidados a participar da pesquisa e informados sobre os objetivos, riscos e benefícios dessa participação. Os critérios de inclusão foram ter idade igual ou superior a 18 anos e exercer a função de cuidador de idosos. Os cuidadores que concordaram em participar assinaram o Termo de Consentimento Livre e Esclarecido (TCLE). Os dados foram coletados através de entrevista com duração de aproximadamente 25 minutos, baseado em um instrumento estruturado, com informações acerca do perfil sociodemográfico, dados das atividades laborais, dos cuidados ofertados aos idosos e a aplicação da escala para rastreio de sofrimento mental comum por meio de índex específico.

\section{Variáveis}

As variáveis descritivas foram distribuídas em tabela e gráfico. Na tabela demonstram-se os aspectos sociodemográficos, econômicos e profissionais dos cuidadores de idosos; faixa etária; sexo; estado civil; escolaridade; rendimento; qualificação profissional e disponibilidade para o cuidado. Em seguida aplicou-se o teste Self Report Questionnaire (SRQ-20) que rastreia a existência de sofrimento mental, através de um instrumento simples, de fácil aplicação e de baixo custo (GONÇALVES et al., 2008). Esse instrumento contém 20 questões relacionadas a desconfortos e enfretamentos vivenciados pelos indivíduos nos últimos 30 dias, com opção de resposta "sim" quando descreve a presença desses enfretamentos e "não" quando há a ausência dos mesmos. As questões abordam problemas relacionados a dores, padrão de sono, estado emocional, funções digestivas, dificuldades nas atividades diárias de vida, tomada de decisões, idéia suicida, dificuldades no trabalho, estado de fadiga e autoestima. O índex do teste corresponde a um resultado maior ou é igual a sete respostas "sim", confirmando assim o rastreio de sofrimento mental leve, vale salientar que o diagnóstico definitivo deve ser realizado por um profissional de saúde capacitado.

Análise

Os instrumentos foram digitados e analisados no programa estatístico 
Statistical Package for the Social Sciences (SPSS), versão 22.0. Desenvolveu-se uma análise descritiva, considerando os números absolutos, frequência e intervalo de confiança $95 \%(I C=95 \%)$ apresentados nas tabelas. Para a variável quantitativa contínua somatório das respostas do SRQ 20, analisou-se média, mediana, mínimo e máximo e desvio padrão (DP).

Aspectos éticos

A presente pesquisa vincula-se a um projeto de pesquisa analisado pelo Comitê de Ética em Pesquisa (CEP) com seres humanos da UFG e, aprovado pelo protocolo no 1.273 .443 , o qual está baseado na Resolução 466/12 do Conselho Nacional de Saúde, que direciona pesquisas envolvendo seres humanos no Brasil.

\section{RESULTADOS E DISCUSSÃO}

Dentre os 14 cuidadores de idosos que compuseram a amostra da presente pesquisa, constatou-se a predominância de idade entre 41-50 anos $(57,1 \%)$; sexo feminino (92,9\%); vivendo sem companheiro (57,1\%); nível de escolaridade até 8 anos (57,1\%); recebe rendimento pelo trabalho de cuidador $(78,6 \%)$ e qualificação profissional de acompanhantes/outros $(78,6 \%)$. Descritos e apresentados na tabela 1.

TABELA 1: Aspectos sociodemográficos, econômicos e profissionais dos cuidadores de idosos. Brasil Central, 2015

\begin{tabular}{lcc}
\hline & $\mathrm{n}(\%)$ & $\mathrm{IC} 95 \%$ \\
\hline Faixa etária & $3(21.4)$ & \\
$30-40$ anos & $\mathbf{8 ( 5 7 . 1 )}$ & $2.0-42.9$ \\
$41-50$ anos & $3(21.4)$ & $0.0-42.9$ \\
$51-60$ anos & $1(7.1)$ & $0.0-21.4$ \\
Sexo & $\mathbf{1 3 ( 9 2 . 9 )}$ & $78.6-100.0$ \\
Masculino & & \\
Feminino & $6(42.9)$ & $14.3-85.7$ \\
Estado civil & $\mathbf{8 ( 5 7 . 1 )}$ & $28.6-85.7$ \\
Solteiro/divorciado/viúvo & $\mathbf{8 ( 5 7 . 1 )}$ & $28.6-85.7$ \\
Vive sem companheiro & $5(35.7)$ & $7.1-64.3$ \\
Escolaridade & $1(7.1)$ & $0.0-21.4$ \\
Até 8 anos & $3(21.4)$ & $0.0-42.9$ \\
Até 11 anos & $\mathbf{1 1}(\mathbf{7 8 . 6 )}$ & $57.1-100.0$ \\
$>11$ anos & & \\
Recebe rendimento & $3(21.4)$ & $7.1-42.9$ \\
Não & $\mathbf{1 1 ( 7 8 . 6 )}$ & $57.1-92.9$ \\
Sim & & \\
Qualificação profissional & $1(7.1)$ & $0.0-21.4$ \\
Técnico/auxiliar de enfermagem & $4(28.6)$ & $7.1-57.1$ \\
Acompanhantes/Outros & $4(28.6)$ & $7.1-57.1$ \\
Disponibilidade para o cuidado & $\mathbf{5 ( 3 5 . 7 )}$ & $14.3-57.1$ \\
Até 6 horas &
\end{tabular}

FONTE: Os autores, (2015). 
Outros autores também relatam a feminização do cuidado. Na Itália, em um estudo de corte transversal com 150 cuidadores de idosos, os resultados apresentaram um perfil de cuidadores predominantemente do sexo feminino $(78 \%)$, idade > de 50 anos (56\%), estado conjugal casado (86\%) e escolaridade: ensino fundamental a médio (42\%) (FAZIO et al., 2015).

No Brasil, várias são as pesquisas que confirmam a feminização no ato de cuidar. Em São Paulo (SP), um estudo com amostra de 100 cuidadores de idosos, resultou em características dos cuidadores de idosos com predominância do sexo feminino (90\%), estado civil casado $(60 \%)$ e com escolaridade de ensino fundamental incompleto (37,3\%) (YAMASHITA et al., 2013).

Quanto a idade, a maior prevalência nesse estudo, foram idades entre 40 e 50 anos, denotando que o adulto maduro é um dos maiores cuidadores de idosos. Em uma pesquisa exploratória-descritiva, com amostra composta por 30 cuidadores, realizada em São Paulo, revelou que a maioria eram mulheres, com idades de 30 a 50 anos (77\%), casadas (66\%) e com ensino fundamental incompleto (26\%) (BALLARIN et al., 2016).

Em relação ao estado civil dos cuidadores, predominou a situação de viverem sem companheiros. Este resultado diverge de outra pesquisa transverval, realizada com cuidadores, revelando que houve uma predominância do sexo feminino (77\%) e com situação conjugal casadas (63\%) (BALLARIN et al., 2016).

Quanto a escolaridade observou-se que $57,1 \%$ possuíam a té oito anos de estudo e que não apresentam qualificação profissional específica para o exercício do cuidado ao idoso $(78,6 \%)$, desempenhando um papel de acompanhante/outros. Um estudo seccional com 40 cuidadores, apontou que, $30 \%$ da amostra apresentavam de cinco a oito anos de estudo, $90 \%$ não tinham formação e capacitação para o cuidado ao idoso e $44,4 \%$ trabalhavam cerca de 24 horas/dia (REIS et al., 2016).

Quanto ao recebimento de rendimento para o exercício do cuidado, o presente estudo demonstrou que $78,6 \%$ dos cuidadores pesquisados recebiam pelo cuidado. Corroborando com o estudo acima citado, uma pesquisa de corte transversal, realizada com 50 cuidadores de idosos em um município do Ceará, apontou que $80 \%$ da amostra possui relação trabalhista para o cuidado e $70 \%$ recebem de um a três salários mínimos. Em relação ao tempo de horas direcionadas aos cuidados para com os idosos, cerca de $70 \%$ confiram que dedicam oito horas/dia, com atividades de cuidado direto aos idosos e cuidados domésticos (ALENCAR et al., 2013).

Em relação as horas dedicadas ao cuidado a pessoa idosa, evidenciou-se nessa pesquisa que a carga horária de trabalho da amostra alcança até 24 horas/dia. Um estudo de natureza epidemiológica, descritiva e transversal, realizado com 240 cuidadores, evidenciou que os participantes do estudo eram na maioria do sexo feminino $(66,2 \%)$, com idade entre 41 a 50 anos $(23 \%)$, vivem com o companheiro $(60,8 \%)$, escolaridade de cinco a oito anos $(46,2 \%)$ e dedicavam para o cuidado cerca de 13 horas ou mais (55,8\%) (LOUREIRA \& FERNANDES, 2015).

Quanto a aplicação do teste Self Report Questionnaire (SRQ-20) mostrado na figura 1, revela os seguintes resultado relacionado a prevalência de respostas "sim": (SRQ1) Tem dores de cabeça frequente (50.0\%); (SRQ2) tem falta de apetite (0.0\%); (SRQ3) dorme mal (28.6\%); (SRQ4) assusta-se com 
facilidade (21.4\%); (SRQ5) tem tremores nas mãos (0.0\%); (SRQ6) sente-se nervoso, tenso ou preocupado (42.9\%); (SRQ7) tem má digestão (35.7\%); (SRQ8) tem dificuldade para pensar com clareza (14.3\%); (SRQ9) tem se sentindo triste ultimamente (21.4\%); (SRQ10) tem chorado mais do que de costume (14.3\%); (SRQ11) encontra dificuldade para realizar com satisfação suas atividades diárias (7.1\%); (SRQ12) tem dificuldade para tomar decisões (21.4\%); (SRQ13) tem dificuldade no serviço (0.0\%); (SRQ14) é incapaz de desempenhar um papel útil em sua vida (14.3\%); (SRQ 15) tem perdido o interesse pelas coisas (7.1\%); (SRQ16) sente-se uma pessoa inútil (0.0\%); (SRQ17) tem tido idéias de acabar com a vida (0.0\%); (SRQ18) sente-se cansado o tempo todo (21.4\%); (SRQ19) cansa-se com facilidade (28.6\%); (SRQ20) tem sensações desagradável no estômago (28.6\%).

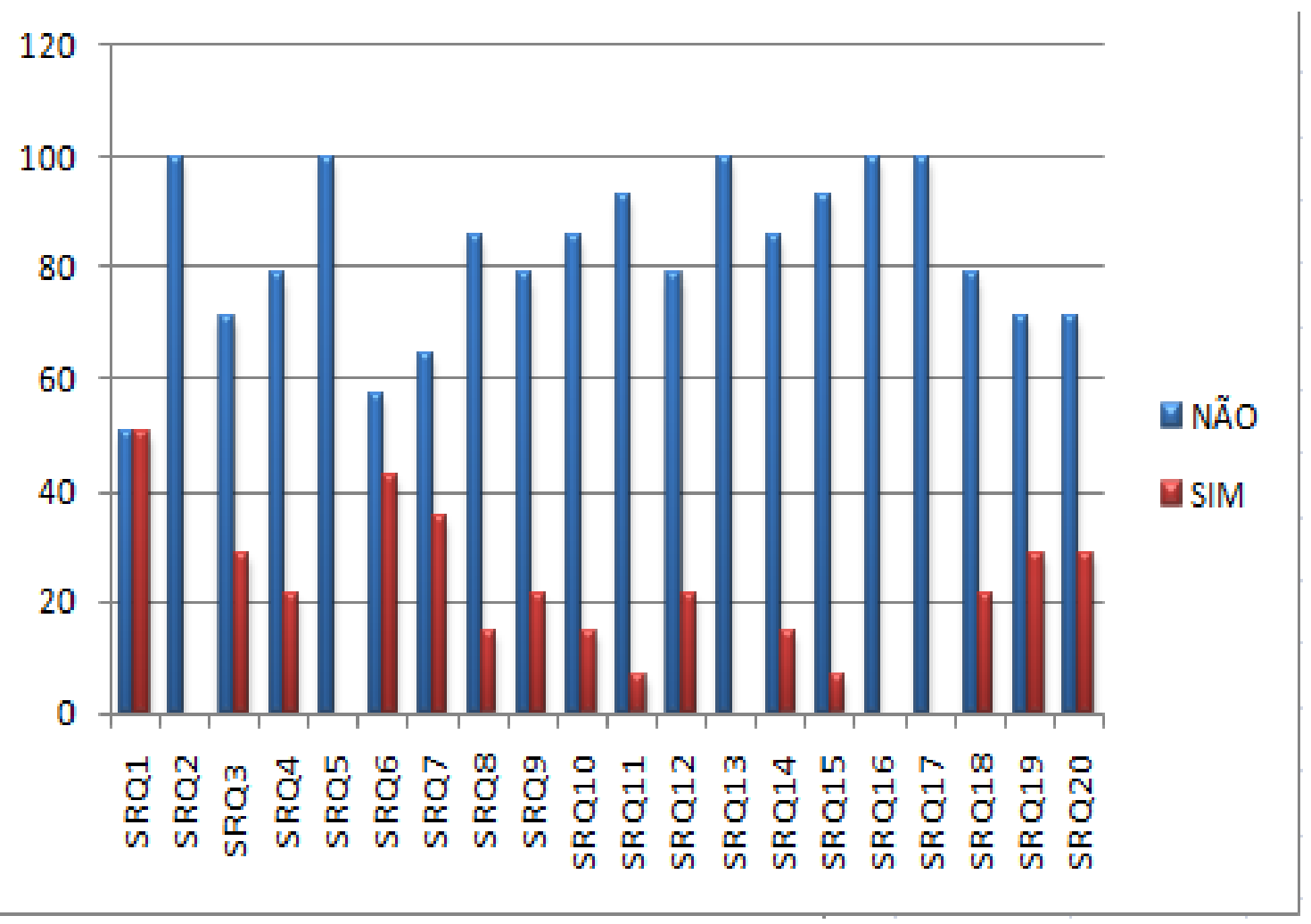

FIGURA 1 - Prevalência das respostas "sim" para as questões do Self-Reporting Questionnaire 20, nos últimos 30 dias.

As questões do SRQ 20 mais prevalentes nesse estudo foram "tem dores de cabeça frequente" $(50.0 \%)$, "sente-se nervoso, tenso ou preocupado" (42.9\%) e "teve má digestão" (35.7\%).

Corroborando com os resultados acima citados, uma pesquisa transversal, realizada com uma amostra de 485 indivíduos, utilizando a aplicação do SRQ-20, apontou que as seguintes questões apresentaram uma prevalência significativa de respostas positivas: "sente nervoso, tenso ou preocupado" $(89,81 \%)$, "cansa-se com facilidade" (77,07\%), "sente-se cansado a todo tempo" (72,61\%), "tem dores de cabeça frequente" $(68,15 \%)$ e "tem sensações desagradáveis no estomago" $(66,88 \%)$ (GONÇALVES et al., 2008).

Quanto à média de TMC encontrada nesse estudo foi de 3,78 , representando 28,6\% (IC95\%: 7.1- 57.1), ou seja, esses indivíduos apresentaram 
escore $\geq 7$, o que indicou a probabilidade para TMC, de acordo com o SRQ 20. Em um estudo realizado no Brasil com 40 cuidadores de idosos, a média do SRQ-20 foi acima de 6,60 pontos, indicando uma alta probabilidade de TMC entre os indivíduos pesquisados (REIS et al., 2016).

Um estudo de corte transversal no estado do Rio Grande do Sul, com 541 indivíduos, demonstrou que as mulheres têm uma prevalência maior do que os homens para TMC, com 46,3\% e 31,6 respectivamente (CARLOTTO et al., 2015). Corroborando com estes autores uma pesquisa desenvolvida na cidade de São Paulo, com 2.366 indivíduos utilizando o SRQ-20 para avaliação da prevalência de TMC, apontou que $64 \%$ eram do sexo feminino, $56,4 \%$ possuíam baixa escolaridade e a média de TMC foi de $43,1 \%$. Em associação com a prevalência de TMC com diversas variáveis, a pesquisa revelou que o sexo feminino, na faixa etária de 40 a 64 anos apresentaram maior chance de TMC e que quanto menor a escolaridade, maior a chance de desenvolver TMC (COUTINHO et al., 2014).

\section{CONCLUSÃO}

A prevalência de TMC entre os cuidadores de idosos vem tornando-se cada vez mais acentuada, provocando um comprometimento na eficiência da assistência a saúde da pessoa idosa. Diante deste contexto faz-se indispensável à realização de novos estudos, que busquem descrever os aspectos sociodemográficos e biopsicossociais dos cuidadores de idosos para oferecer fomento na criação e implementação de políticas públicas que garantam a qualidade na atenção a saúde e qualidade de vida da pessoa idosa, bem como de seu cuidador.

\section{REFERÊNCIAS}

ALENCAR, L.S. de; SARAIVA, J.M.; ALENCAR, J.S. Educação Profissional Cidadã: ampliando a concepção dos cuidadores(as) de idosos(as) acerca do processo de envelhecimento para além das práticas de cuidado. Revista Kairós Gerontologia, v.16, n.3, p. 103-116, 2013.

ANGST, J.; PAKSARIAN, D.; CUI, L.; MERIKANGAS, K.R.; HENGARTNER, M.P.; GROSS, V.A.; ROSSLER, W. The epidemiology of common mental disorders from age 20 to 50: results from the prospective Zurich cohort Study. Epidemiology and Psychiatric Sciences, v.25, n.1, p.24-32, fev., 2015.

BALLARIN, M.L.G.S.; BENEDITO, A.C.; KRON, C.A.; CHRISTOVAM, D. Sociodemographic profile and burden of informal caregivers of patients assisted in occupational therapy outpatient clinic. Cad. Ter. Ocup. UFSCar, v. 24, n. 2, p. 315- 321, 2016.

BATISTA, A.S.; ARAÚJO, A.B. Intimidade e Mercado: o cuidado de idosos em Instituições de Longa Permanência para Idosos. Rev. soc. estado, v.26, n.1, p.175- 95, 2011.

BAXTER, A.J.; PATTON, G.; SCOTT, K.M.; DEGENHARDT, L.; WHITEFORD, H.A. Global Epidemiology of Mental Disorders: What Are We Missing?. Plos one, v.24, p. 1-10, 2013. 
BESSANT, M.; EKERS, D. Common mental health disorders. Nurs Times, v.107, n. 26, p.5-11, jul., 2011.

CARLOTTO, M.S.; BARCINSKI, M.; FONSECA, R. Transtornos mentais comuns e associação com variáveis sociodemográficas e estressores ocupacionais: uma análise de gênero. Estud. pesqui. psicol., v.15, n.3, p.1006-1026, 2015.

COUTINHO, L.M.S.; MATIJASEVICH, A.; SCAZUFCA, M.; MENEZES, P.R. Prevalência de transtornos mentais comuns e contexto social: análise multinível do São Paulo Ageing \& Health Study (SPAH). Cad. Saúde Pública, v.30, n.9, p.1875-1883, set, 2014.

FAZIO, P. de; CIAMBRONE, P.; CERMINARA, G.; BARBUTO, E.; BRUNI, A.; GENTILE, $P$. et al. Depressive symptoms in caregivers of patients with dementia: demographic variables and burden. Clinical Interventions in Aging, v.10, p.1085-1090, 2015.

GONÇALVES, D.M.; STEIN, A.T.; KAPCZINSKI, F. Avaliação de desempenho do Self-Reporting Questionnaire como instrumento de rastreamento psiquiátrico: um estudo comparativo com o Structured Clinical Interview for DSM-IV-TR. Cad. Saúde Pública, v.24, n.2, p.380-390, fev., 2008.

GRATÃO, A. C. M.; TALMELLI, L. F. S.; FIGUEIREDO, L. C.; ROSSET, I.; FREITAS, C. P.; RODRIGUES, R. A. P. Dependência funcional de idosos e a sobrecarga do cuidador. Revista da Escola de Enfermagem da USP, São Paulo, v. 47, n. 1, p. 137-144, 2013.

GRATÃO, A.C.M.; COSTA, A.C.da; DINIZ, M.A.A.; NERI, K.H.; MELO, B.R. de S. Condições de saúde de idosos e cuidadores em uma instituição de longa permanência para idosos. Rev enferm UFPE on line, Recife,v.9, $n$ 3, p.7562-7571, abr., 2015.

HARDING, T.W.; ARANGO, M.V.; BALTAZAR, J. ;CLIMENT, C.E.; IBRAHIM, H.H.A.; IGNACIO, L.L.; MURTHY, R.S. \& WIG, N.N. Mental Disorders in primary health care: a study of their frequency and diagnosis in four development contries. Psychological Medicine, v.10, p. 231-241, 1980.

LOUREIRA, L.S.N.; FERNANDES, M.G.M. Perfil do cuidador familiar de idosos dependentes em convívio domiciliar. Journal of Research Fundamental Care Oline, v.7, p. 145-154, dez., 2015.

MARI, J.; WILLIANS, P.A . A validity study of a psychiatric screening questionnaire (SRQ-20) in primary care in the city of São Paulo. Brit. J. Psychiatry, v.148, p. 23- 26, 1986.

MEDRONHO, R. de A.; BLOCH, K.V.; LUIZ, R.R.; WERNECHE, G.L. Epidemiologia. São Paulo: Editora Atheneu, 2009.

MOREIRA, J.K.P.; BANDEIRA, M.; CARDOSO, C.S.; SCALON, L.D. Prevalência de transtornos mentais comuns e fatores associados em uma população assistida por equipes do Programa Saúde da Família. Jornal Bras. Psiquiatria, v.60, n.3, p.221- 
6, 2011.

ORGANIZAÇÃO MUNDIAL DA SAÚDE. CID-10 Classificação Estatística Internacional de Doenças e Problemas Relacionados à Saúde. 10a rev. São Paulo: Universidade de São Paulo; 1997. Disponível em: <www.datasus.gov.br/cid10/download.htm>. Acesso em: 10 set. 2016.

PRINCE, M.; PATEL, V.; SAXENA, S.; MAJ, M.; MASELKO, J.; PHILLIPS, M.R.; RAHMAN, A. No health without mental health. Lancet, v.370, p.859-877, set., 2007.

ROSA, P.V.; GLOCK, L.; BERLEZI, E.M.; ROSSATO, D.D.; ROSA, L.H.T. Perfil dos idosos residentes em instituições de longa permanência da região sul do país. Rev Bras Ciênc Envelhec Hum., v.8, n.1, p. 38-47, 2011.

REIS, L.A. dos; SANTOS, K.T. dos; GOMES, N.P.; REIS, L.A. Determinantes da sobrecarga e desconforto emocional em cuidadores de idosos. Revista Enfermagem Contemporânea, v.5, n.1, p.59-67, jan./jun., 2016.

SKAPINAKIS, P.; BELLOS, S.; KOUPIDIS, S.; GRAMMATIKOPOULOS, I.; THEODORAKIS, P.N.; MAVREAS, V.Prevalence and sociodemographic associations of common mental disorders in a nationally representative sample of the general population of Greece. BMC Psychiatry, v.13, p. 1-14, 2013.

STEEL, Z.; MARNANE, C.; IRANPOUR, C.; CHEY, T.; JACKSON, J.W.; PATEL, V. ; SILOVE, D. The global prevalence of common mental disorders: a systematic review and meta-analysis 1980-2013. International Journal of Epidemiology, v. 43, n. 2, p.476-493, 2014.

YAMASHITA, C. H.; AMENDOLA, F.; GASPAR, J.C.; ALVARENGA, M.R.M.; OLIVEIRA, M.A. de C. Associação entre o apoio social e o perfil de cuidadores familiares de pacientes com incapacidades e dependência. Rev Esc Enferm USP, v.47, n.6, p.1359-1366, 2013. 